\title{
Vibration of Elastic Functionally Graded Thick Rings
}

\author{
Guang-Hui Xu, ${ }^{1,2}$ Huai-Wei Huang, ${ }^{1}$ and Yong-Qiang Zhang ${ }^{1}$ \\ ${ }^{1}$ School of Civil Engineering and Transportation, South China University of Technology, Guangzhou, Guangdong 510640, China \\ ${ }^{2}$ Guangzhou Institute of Measuring and Testing Technology, Guangzhou, Guangdong 510663, China \\ Correspondence should be addressed to Huai-Wei Huang; cthwhuang@scut.edu.cn
}

Received 8 November 2016; Accepted 5 January 2017; Published 16 February 2017

Academic Editor: Toshiaki Natsuki

Copyright (C) 2017 Guang-Hui Xu et al. This is an open access article distributed under the Creative Commons Attribution License, which permits unrestricted use, distribution, and reproduction in any medium, provided the original work is properly cited.

\begin{abstract}
The free vibration behaviors of functionally graded rings were investigated theoretically. The material graded in the thickness direction according to the power law rule and the rings were assumed to be in plane stress and plane strain states. Based on the first-order shear deformation theory and the kinetic relation of von Kárman type, the frequency equation for free vibration of functionally graded ring was derived. The derived results were verified by those in literatures which reveals that the present theory can be appropriate to predict the free vibration characteristics for quite thick rings with the radius-to-thickness ratio from 60 down to 2.09. Comparison between the plane stress case and the plane strain case indicates a slight difference. Meanwhile, the effects of the structural dimensional parameters and the material inhomogeneous parameter are examined. It is interesting that the value of the logarithmic form of vibration frequency is inversely proportional to the logarithmic form of the radius-to-thickness ratio or the mean radius.
\end{abstract}

\section{Introduction}

Circular ring structures are widely employed in industrial systems. Their applications can be found in planetary gears, rotors, gyroscopic actuators, measuring instruments, and hollow axle used on subway and high-speed trains. Their mechanical performances have been investigated extensively, among which the vibration characteristics are one of the most important aspects [1-7].

Matsunaga [1] presented an approximate theory to study the effects of higher-order deformations on natural frequencies and buckling critical loads of a thick circular ring with rectangular cross-sections. Wong et al. [2] considered inplane vibration problem for thermoelastic damping rings. Lacarbonara et al. [7] analyzed flexural vibration problem of elastic circular rings considering nonlinearity of structural deformation.

The new emergence of ceramic/metallic compound functionally graded materials (FGMs) has attracted tremendous research interests on vibration characteristics for their plate and shell structures [8-10]. For FGM rings, Filipich et al. [11] introduced a general model for FGM thick arches or rings under transient forced vibration conditions. Wang and Luo
[12] presented an exact solution for the electromechanical vibration of FGM piezoelectric ring transducers. Among these works, the free vibration behaviors of FGM circular rings under either the plane stress or plane strain states have yet not been investigated.

In this paper, free vibration characteristics of FGM ring under either the plane stress or plane strain states were investigated by using the first-order shear deformation theory. The accuracy of the present theory was well verified through the free vibration frequencies of homogeneous rings. It reveals that the present theory can be appropriate to predict the free vibration characteristics for the quite thick ring with the radius-to-thickness ratio from 60 down to 2.09. Meanwhile, the effects of the structural dimensional parameters and the material inhomogeneous parameter are examined.

\section{Formulation}

The geometry and the coordinate system are illustrated in Figure 1, in which the coordinate system is represented by $r-\theta-x . R$ is the mean radius, and $h$ and $b$ are, respectively, the thickness in $r$ and $x$ directions. $z$ indicates the distance 


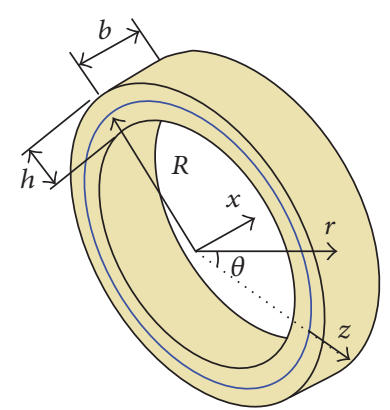

FIgURE 1: The geometry and the coordinate system of FGM rings.

measure from the geometrical middle plane of the ring and we have $z=r-R$.

FGMs are usually prepared by mixing ceramic and metallic constituents. The volume fraction of one of the constituents $f_{i}(i=$ cor $\mathrm{m})$ through the thickness $h$ submits to a power law rule [8] and the elastic material properties $P(z)$, representing the elastic modulus $E(z)$ and the poison ratio $v(z)$, can be expressed as the weight average of their constituents.

$$
\begin{aligned}
f_{i} & =\left(0.5+\frac{z}{h}\right)^{k}, \\
f_{\mathrm{c}}+f_{\mathrm{m}} & =1, \\
P & =P_{\mathrm{m}} f_{\mathrm{m}}+P_{\mathrm{c}} f_{\mathrm{c}},
\end{aligned}
$$

where the subscripts $\mathrm{c}$ and $\mathrm{m}$ denote, respectively, the ceramic and metallic constituents and $f$ and $P$ are the volume fraction and the material properties. Herein, the FGM ring with metallic internal surface is named Type I configuration, in which $i=\mathrm{c}$, while the FGM ring with ceramic internal surface is named Type II configuration, in which $i=\mathrm{m}$.

For the plane strain rings, $\varepsilon_{x x}=0$, and for the plane stress rings, $\sigma_{x x}=0$. Thus, the general constitutive relation of FGM rings can be obtained as

$$
\left[\begin{array}{c}
\sigma_{x x} \\
\sigma_{\theta \theta} \\
\tau_{r \theta}
\end{array}\right]=\left[\begin{array}{ll}
C_{12} & C_{13} \\
C_{22} & C_{23} \\
C_{32} & C_{33}
\end{array}\right]\left[\begin{array}{l}
\varepsilon_{\theta \theta} \\
\gamma_{r \theta}
\end{array}\right],
$$

where

$$
\left[\begin{array}{ll}
C_{12} & C_{13} \\
C_{22} & C_{23} \\
C_{32} & C_{33}
\end{array}\right]=\left[\begin{array}{cc}
\frac{\vartheta E \nu}{\left(1-\nu^{2}\right)} & 0 \\
(1-\vartheta) E+\frac{\vartheta E}{\left(1-\nu^{2}\right)} & 0 \\
0 & \frac{E}{[2(1+\nu)]}
\end{array}\right]
$$

and $\vartheta=0,1$ respectively, indicate the plane stress state and the plane strain state.
The kinetic relation of von Kárman type reads in the following form [14] with the subscript comma representing partial derivative.

$$
\begin{aligned}
& \varepsilon_{\theta \theta}=\frac{\left(V_{, \theta}+W\right)}{r}+\frac{\left(W_{, \theta}-V\right)^{2}}{\left(2 r^{2}\right)}, \\
& \gamma_{r \theta}=\frac{\left(W_{, \theta}-V\right)}{r}+V_{, r},
\end{aligned}
$$

in which, the circumferential displacement $V(\theta, z, t)$ and the radial displacement $W(\theta, z, t)$ can be expressed as the following form according to the first-order shear deformation theory [15]. $t$ is time.

$$
\begin{aligned}
V & =v+z \varphi, \\
W & =w,
\end{aligned}
$$

where $v(\theta, t), w(\theta, t)$, and $\varphi(\theta, t)$ are, respectively, the circumferential, radial, and angle displacements on the geometrical middle plane. Generally, $r \approx R, z / R \ll 1$; thus we have

$$
\left[\begin{array}{c}
\varepsilon_{\theta \theta} \\
\gamma_{r \theta}
\end{array}\right]=\left[\begin{array}{c}
\varepsilon_{\theta \theta 0} \\
\gamma_{r \theta 0}
\end{array}\right]+z\left[\begin{array}{c}
\varepsilon_{\theta \theta 1} \\
0
\end{array}\right]
$$

where

$$
\begin{aligned}
& \varepsilon_{\theta \theta 0}=\frac{\left(v_{, \theta}+w\right)}{R}+\frac{\left[\left(w_{, \theta}-v\right) / R\right]^{2}}{2}, \\
& \varepsilon_{\theta \theta 1}=\frac{\varphi_{, \theta}}{R}, \\
& \gamma_{r \theta 0}=\varphi+\frac{\left(w_{, \theta}-v\right)}{R} .
\end{aligned}
$$

The internal force $N$ and shear force $Q$, as well as the internal moment $M$, can be obtained as follows:

$$
\left[\begin{array}{lll}
N & M & Q
\end{array}\right]^{\mathrm{T}}=\int_{-h / 2}^{h / 2}\left[\begin{array}{llll}
\sigma_{\theta \theta} & z \sigma_{\theta \theta} & K_{s} \tau_{r \theta}
\end{array}\right]^{\mathrm{T}} \mathrm{d} z
$$

where $K_{s}=5 / 6$ is the shear correction factor. Submitting (6) into (2) and then into (8) derives

$$
\left[\begin{array}{l}
N \\
M \\
Q
\end{array}\right]=\left[\begin{array}{lll}
C_{220} & C_{221} & C_{230} \\
C_{221} & C_{222} & C_{231} \\
C_{320} & C_{321} & C_{330}
\end{array}\right]\left[\begin{array}{c}
\varepsilon_{\theta \theta 0} \\
\varepsilon_{\theta \theta 1} \\
K_{s} \gamma_{r \theta 0}
\end{array}\right],
$$

where $C_{i j l}=\int_{-h / 2}^{h / 2} z^{l} C_{i j} \mathrm{~d} z(i, j=2,3, l=0,1,2)$.

According to the principle of virtual displacement, the virtual potential energy $\delta U$ should vanish at the equilibrium state of the system.

$$
\delta U=\delta U_{\mathrm{in}}-\delta U_{\mathrm{ke}}=0
$$


in which

$$
\begin{aligned}
& \delta U_{\mathrm{in}}=\int_{0}^{b} \int_{0}^{2 \pi} \int_{-h / 2}^{h / 2}\left(\sigma_{\theta \theta} \delta \varepsilon_{\theta \theta}+K_{s} \tau_{r \theta} \delta \gamma_{r \theta}\right) \mathrm{d} z R \mathrm{~d} \theta \mathrm{d} x \\
& \delta U_{\mathrm{ke}}=\int_{0}^{b} \int_{0}^{2 \pi} \int_{-h / 2}^{h / 2}\left[\rho(R+z) \times\left(w_{, t} \delta w_{, t}+v_{, t} \delta v_{, t}+\frac{h^{2}}{12} \varphi_{, t} \delta \varphi_{, t}\right)\right] \mathrm{d} z \mathrm{~d} \theta \mathrm{d} x,
\end{aligned}
$$

where $\rho(z)$ is the density of FGMs. After integration, we have

$$
\begin{aligned}
& \delta U_{\mathrm{in}}=b R \int_{0}^{2 \pi}\left(N \delta \varepsilon_{\theta \theta 0}+M \delta \varepsilon_{\theta \theta 1}+Q \delta \gamma_{r \theta 0}\right) \mathrm{d} \theta, \\
& \delta U_{\mathrm{ke}}=b \widehat{\rho} \int_{0}^{2 \pi}\left(w_{, t} \delta w_{, t}+v_{, t} \delta v_{, t}+\frac{h^{2} \varphi_{, t} \delta \varphi_{, t}}{12}\right) \mathrm{d} \theta,
\end{aligned}
$$

where $\hat{\rho}=\int_{-h / 2}^{h / 2} \rho(R+z) \mathrm{d} z$. Then (10) turns into

$$
\begin{aligned}
\int_{0}^{2 \pi} & {\left[R\left(N \delta \varepsilon_{\theta \theta 0}+M \delta \varepsilon_{\theta \theta 1}+Q \delta \gamma_{r \theta 0}\right)\right.} \\
& \left.-\hat{\rho}\left(w_{, t} \delta w_{, t}+v_{, t} \delta v_{, t}+\frac{h^{2} \varphi_{, t} \delta \varphi_{, t}}{12}\right)\right] \mathrm{d} \theta=0 .
\end{aligned}
$$

The above relation should hold for arbitrary virtual displacements $\delta w, \delta v$, and $\delta \varphi$ which reaches the dynamic equilibrium equation set of FGM rings, by combining the virtual form of $\varepsilon_{\theta \theta 0}, \varepsilon_{\theta \theta 1}$, and $\gamma_{r \theta 0}$ with (11) and then utilizing the procedure of part integration.

$$
\begin{aligned}
\frac{\left[N\left(w_{, \theta}-v\right)\right]_{, \theta}}{R}-N+Q_{, \theta} & =\hat{\rho} w_{, t t}, \\
N_{, \theta}+Q+\frac{N\left(w_{, \theta}-v\right)}{R} & =\hat{\rho} v_{, t t}, \\
M_{, \theta}-R Q & =\frac{h^{2} \widehat{\rho} \varphi_{, t t}}{12} .
\end{aligned}
$$

With the aid of (9), the above dynamic equilibrium equations can be expressed by displacement components.

$$
\begin{aligned}
& C_{330} K_{s}\left(R \varphi+w_{, \theta}-v\right)_{, \theta}-C_{220}\left(w+v_{, \theta}\right) \\
& \quad-C_{221} \varphi_{, \theta}=R \widehat{\rho} w_{, t t}, \\
& C_{330} K_{s}\left(R \varphi+w_{, \theta}-v\right)+C_{220}\left(w+v_{, \theta}\right)_{, \theta} \\
& \quad+C_{221} \varphi_{, \theta \theta}=R \widehat{\rho} v_{, t t}, \\
& -C_{330} K_{s} R\left(R \varphi+w_{, \theta}-v\right)+C_{221}\left(w+v_{, \theta}\right)_{, \theta} \\
& \quad+C_{222} \varphi_{, \theta \theta}=\frac{R h^{2} \hat{\rho} \varphi_{, t t}}{12} .
\end{aligned}
$$

\section{Solving of the Problem}

Herein, the vibration modal functions are set as the following form:

$$
\begin{aligned}
w & =\xi_{w} \sin (n \theta) e^{i \omega t}, \\
v & =\xi_{\nu} \cos (n \theta) e^{i \omega t}, \\
\varphi & =\xi_{\varphi} \cos (n \theta) e^{i \omega t},
\end{aligned}
$$

where $\xi_{w}, \xi_{v}$, and $\xi_{\varphi}$ are the unknown amplitudes and $n$ is the wave number. $\omega=2 \pi f$ is the angle frequency.

By substituting (16) into (15), one obtains

$$
\left\{\left[\begin{array}{lll}
\chi_{11} & \chi_{12} & \chi_{13} \\
\chi_{12} & \chi_{22} & \chi_{23} \\
\chi_{13} & \chi_{23} & \chi_{33}
\end{array}\right]+R \omega^{2} \widehat{\rho}\left[\begin{array}{ccc}
1 & 0 & 0 \\
0 & 1 & 0 \\
0 & 0 & \frac{h^{2}}{12}
\end{array}\right]\right\}\left[\begin{array}{l}
\xi_{w} \\
\xi_{v} \\
\xi_{\varphi}
\end{array}\right]=0
$$

in which

$$
\begin{aligned}
& \chi_{11}=-C_{220}-n^{2} C_{330} K_{s}, \\
& \chi_{12}=n\left(C_{220}+C_{330} K_{s}\right), \\
& \chi_{13}=n\left(C_{221}-R C_{330} K_{s}\right), \\
& \chi_{22}=-n^{2} C_{220}-C_{330} K_{s}, \\
& \chi_{23}=-n^{2} C_{221}-R C_{330} K_{s}, \\
& \chi_{33}=-n^{2} C_{222}-R^{2} C_{330} K_{s} .
\end{aligned}
$$

From (17), the zero value of the determinant of the coefficient matrix leads to the frequency equation for FGM rings, a six-degree polynomial, which can be used to solve for the free vibration frequency of FGM rings

$$
\sum_{j=0}^{3} \gamma_{j} \omega^{2 j}=0 .
$$

\section{Verification}

To verify the present theoretical results, Table 1 lists comparisons of the present theoretical results with the theoretical and experimental results in literatures with regard to homogeneous rings and the results from ANSYS simulation.

For moderate FGM rings with $R / h=60$, the present plane stress results are in excellent agreement with the results from ANSYS, and the present plane strain results are well verified by the results from Bisegna and Caruso [3]. For thick FGM rings with $R / h=2.09$, the present plane strain results are close to the theoretical predictions of Kirkhope [13] and the experimental results of Kuhl listed in [13]. The maximal difference of the present plane strain results from the experimental ones is only $-2.2 \%$. Since both the cases $R / h=60$ and 2.09 indeed stand for a moderate thick and even quite thick rings, the present theories are appropriate to predict the free vibration characteristics for FGM rings of thick configuration. 
TABLE 1: Verification of free vibration frequency $[\mathrm{Hz}]$.

\begin{tabular}{|c|c|c|c|c|}
\hline \multirow{2}{*}{$n$} & \multirow{2}{*}{ Bisegna and Caruso [3] } & \multirow{2}{*}{$\begin{array}{c}\text { ANSYS } \\
\text { Plane stress }\end{array}$} & \multicolumn{2}{|c|}{ The present theory } \\
\hline & & & Plane stress & Plane strain \\
\hline 2 & 36.78 & 35.10 & 35.08 & 36.77 \\
\hline 3 & 104.03 & 99.29 & 99.19 & 103.98 \\
\hline 4 & 199.46 & 190.42 & 190.13 & 199.3 \\
\hline 5 & 322.57 & 308.05 & 307.35 & 322.16 \\
\hline \multirow{2}{*}{$n$} & \multirow{2}{*}{ Exp. of Kuhl [13] } & \multirow{2}{*}{ Kirkhope [13] } & \multicolumn{2}{|c|}{ The present theory } \\
\hline & & & Plane stress & Plane strain \\
\hline 2 & 7635 & 7527 & 7300 & 7566 \\
\hline 3 & 19060 & 19122 & 18269 & 18830 \\
\hline 4 & 32150 & 32529 & 30898 & 31694 \\
\hline 5 & 46050 & 46614 & 114803 & 45246 \\
\hline 6 & 60400 & 60857 & 57997 & 59068 \\
\hline 7 & 74200 & 75045 & 71828 & 72962 \\
\hline 8 & 88000 & 89104 & 85673 & 86838 \\
\hline
\end{tabular}
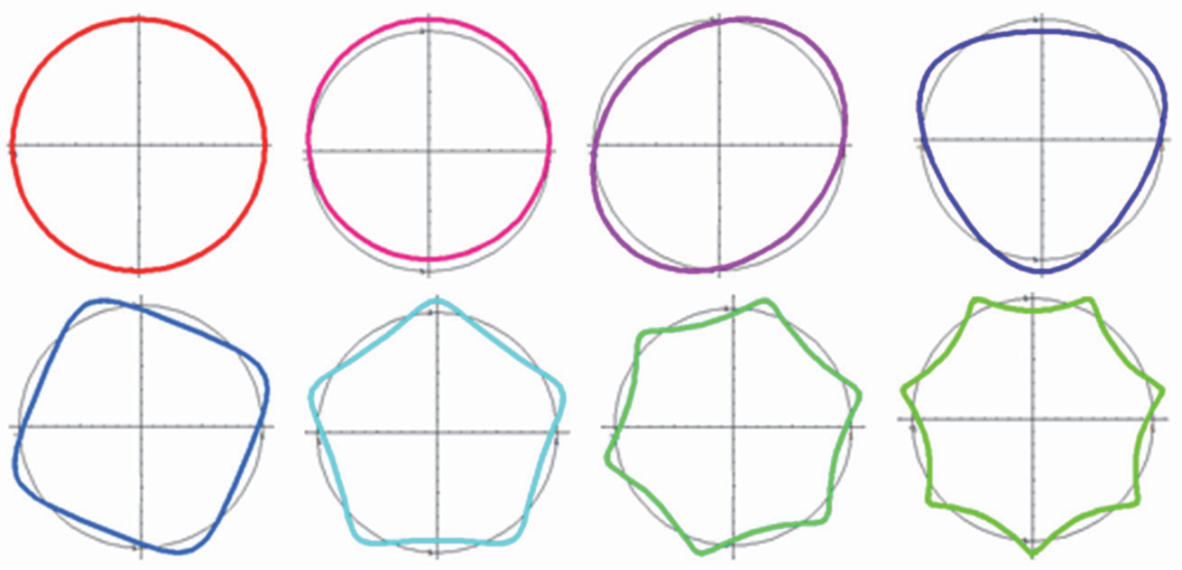

FIGURE 2: The vibration mode of FGM rings $(n=0,1,2, \ldots, 7)$.

\section{Result Analyses}

The following calculations are presented for rings made from Si3N4/SUS304 FGMs, with the material properties given as

$$
\begin{aligned}
\rho_{\mathrm{m}} & =8166 \mathrm{~kg} / \mathrm{m}^{3}, \\
E_{\mathrm{m}} & =107 \mathrm{GPa}, \\
\nu_{\mathrm{m}} & =0.34, \\
\rho_{\mathrm{c}} & =2370 \mathrm{~kg} / \mathrm{m}^{3}, \\
E_{\mathrm{c}} & =375 \mathrm{GPa}, \\
\nu_{\mathrm{c}} & =0.14 .
\end{aligned}
$$

For briefness, the calculation parameters are listed in all the following figures as well. Figure 2 shows the vibration modes under different values of the modal parameter $n$. In the figure, $n=0$ represents an undeformed ring, and $n=1$ indicates a rigid body displacement. For Type I FGM rings, Figure 3 shows the effect of the modal parameter on the

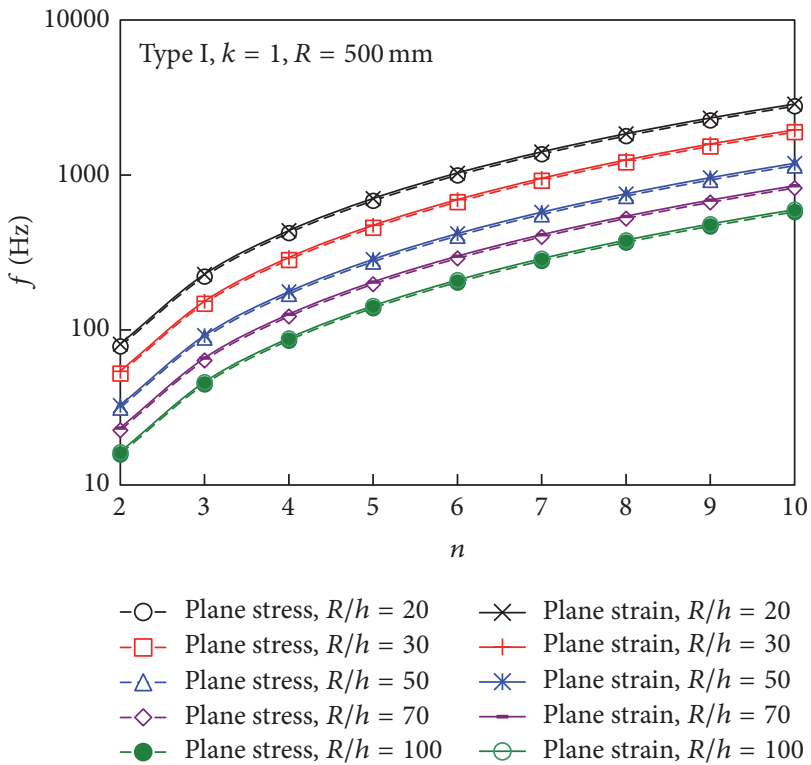

FIGURE 3: Effect of the modal parameter on vibration frequency. 


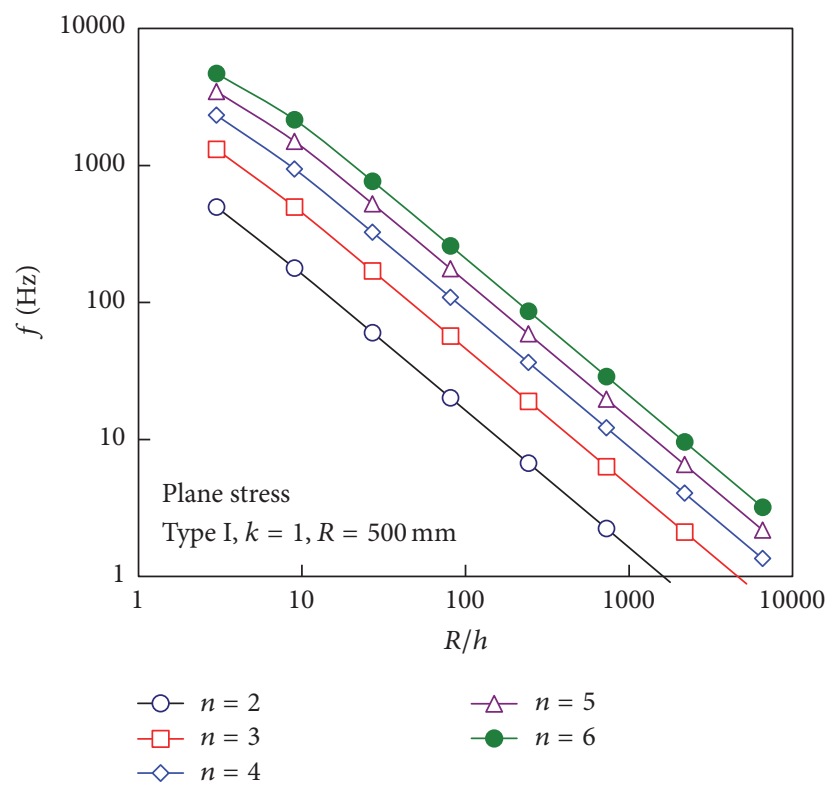

(a)

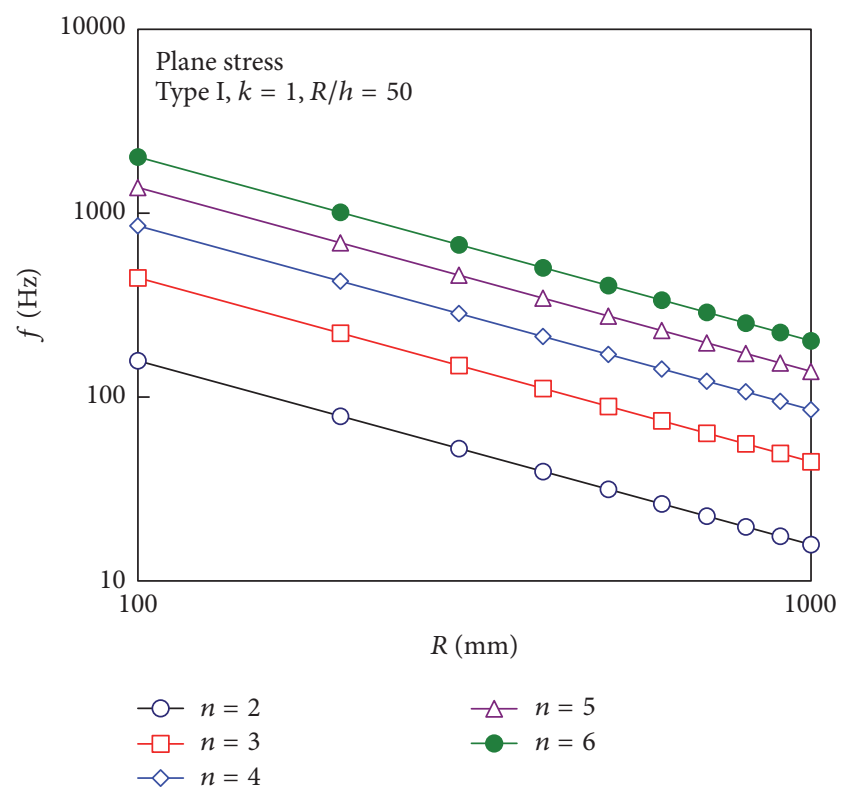

(b)

FIGURE 4: Dimensional effect on vibration frequency.

free vibration frequency $f$. The vibration frequency (plotted in the logarithmic form) increases dramatically with the increasing value of $n$. The lowest value of vibration frequency generally corresponds to a low order vibration mode, that is, $n=2$, which is the first concern for engineering structures. Comparison between the plane stress and the plane strain cases reveals that the vibration frequency in the plane strain case is slightly higher than that in the plane stress case.

The changing rules of the vibration frequency versus different values of $R / h$ are illuminated in Figure 4(a), where both the horizontal and the longitudinal coordinates are given in the logarithmic form. It is obvious that the value of the logarithmic $f$ is inversely proportional to the logarithmic value of $R / h$. Similarly, when the value of $R / h$ is set to be 50, Figure 4(b) shows that the logarithmic value of $f$ is also inversely proportional to the logarithmic value of $R$.

By considering both Type I and Type II configurations of FGM rings, Figure 5 provides the relation of the vibration frequency versus the inhomogeneous parameter $k$. For Type I FGM rings, the vibration frequency increases with the rising value of $k$, while, for Type II ones, there is an opposite tendency.

As the aforementioned, the vibration frequency of FGM rings in the plane strain case is usually larger than that in the plane stress case. To quantitatively determine the deviations between them, Figure 6 plots the differences versus the dimensional parameter $R / h$, the inhomogeneous parameter, and the modal parameter as well. As shown, the differences under different value of $n$ tend to be a constant $3.4 \%$ when $R / h \geq 50$. In the case of thick rings with $3 \leq R / h<50$, the differences range from $2.5 \%$ to $3.5 \%$. The difference between them increases with the rising value of $k$. For a nearly pure ceramic ring with $k=0.001$, it tends to be $1 \%$, but for a nearly pure metallic ring with $k=1000$, it is up to $6.2 \%$.

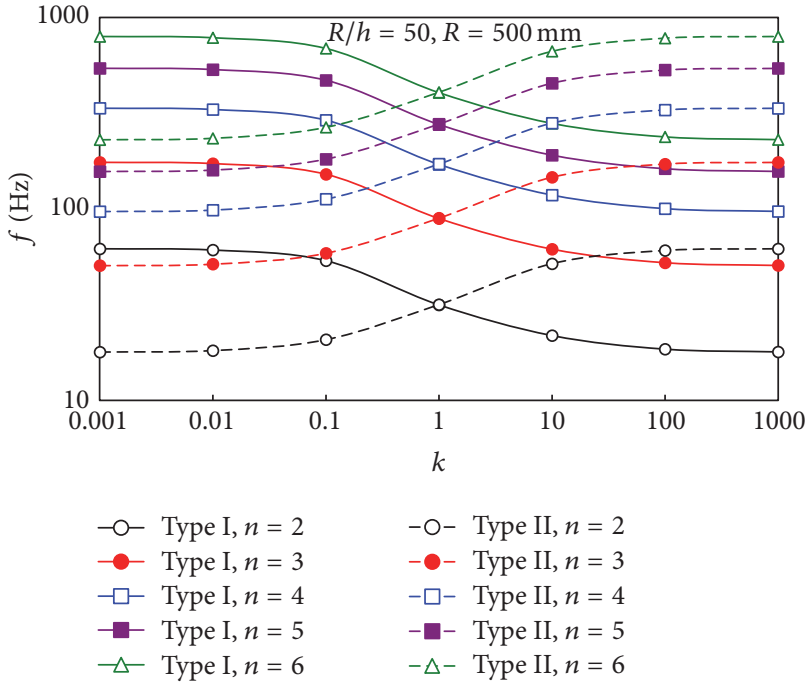

FIGURE 5: Effect of the inhomogeneous parameter on the vibration frequency for the plane stress case.

\section{Concluding Remark}

This paper is addressed for free vibration characteristics of FGM rings under either the plane stress or the plane strain cases; the frequency equation is derived based on the firstorder shear deformation theory and the kinetic relation of von Kárman type. Results well agree with those in literatures and ANSYS simulation. It is also shown that the vibration frequency of FGM rings under the plane strain case is slightly higher than that under the plane stress case, and it increases dramatically with the rising modal parameter. Under a given value of radius, the vibration frequency decreases sharply 


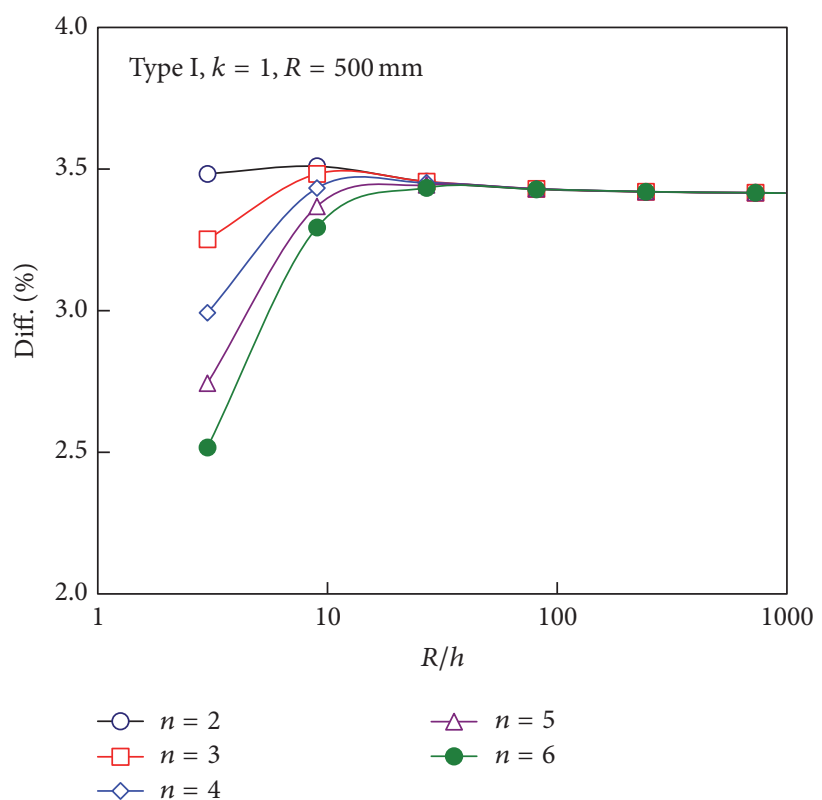

(a)

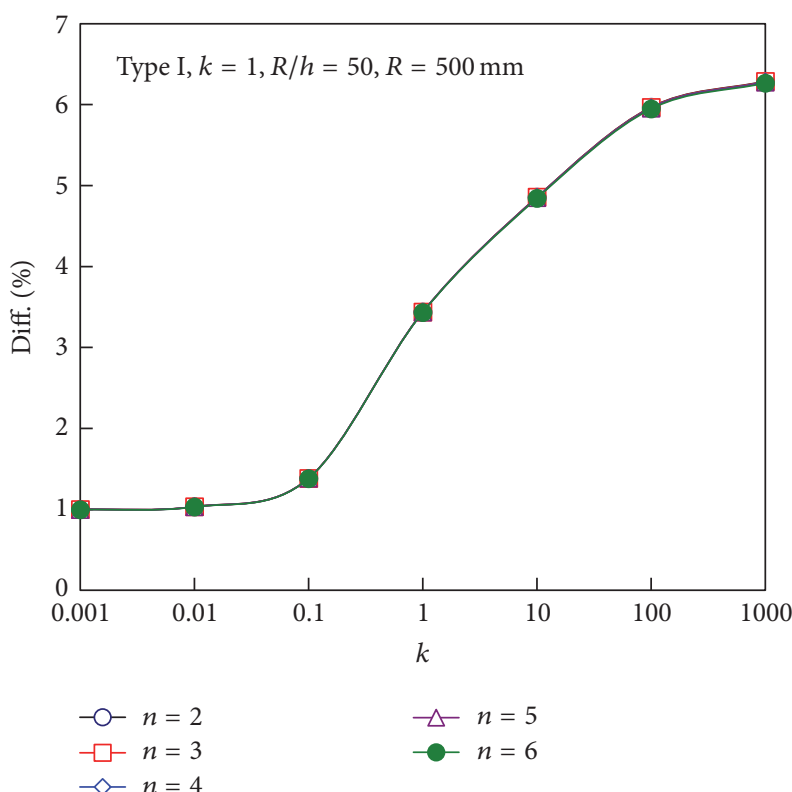

(b)

FIGURE 6: Differences of the vibration frequencies between the plane strain and the plane stress cases.

with the increase of radius-to-thickness ratio, and the value of the logarithmic vibration frequency is inversely proportional to the logarithmic value of radius-to-thickness ratio. Under a given value of the radius-to-thickness ratio, the vibration frequency also decreases sharply with the increase of the mean radius. For Type I FGM rings, the vibration frequency increases with the rising value of the inhomogeneous parameter, while, for Type II ones, there is an opposite tendency.

\section{Competing Interests}

The authors declare that they have no competing interests.

\section{Acknowledgments}

This work was supported by the National Natural Science Foundation of China (Grant no. 11402093), State Key Laboratory for Strength and Vibration of Mechanical Structures (Grant no. SV2016-KF-08), the Fundamental Research Funds for the Central Universities, SCUT (Grant no. 2015ZZ130), and the Science and Technology Program of Guangzhou (Grant no. 201607010282).

\section{References}

[1] H. Matsunaga, "Effects of higher-order deformations on inplane vibration and stability of thick circular rings," Acta Mechanica, vol. 124, no. 1-4, pp. 47-61, 1997.

[2] S. J. Wong, C. H. J. Fox, and S. McWilliam, "Thermoelastic damping of the in-plane vibration of thin silicon rings," Journal of Sound and Vibration, vol. 293, no. 1-2, pp. 266-285, 2006.

[3] P. Bisegna and G. Caruso, "Frequency split and vibration localization in imperfect rings," Journal of Sound and Vibration, vol. 306, no. 3-5, pp. 691-711, 2007.
[4] D. Zhou, Y. K. Cheung, and S. H. Lo, "3-D vibration analysis of circular rings with sectorial cross-sections," Journal of Sound and Vibration, vol. 329, no. 9, pp. 1523-1535, 2010.

[5] L. Wang, Y. Lu, Y. Xiang, L. Qin, and D. Cai, "Vibration analysis for piezoceramic ring," Ceramics International, vol. 39, no. 1, pp. S739-S742, 2013.

[6] D. Beli, P. B. Silva, and J. R. De Francą Arruda, "Vibration analysis of flexible rotating rings using a spectral element formulation," Journal of Vibration and Acoustics, Transactions of the ASME, vol. 137, no. 4, Article ID 041003, 11 pages, 2015.

[7] W. Lacarbonara, A. Arena, and S. S. Antman, "Flexural vibrations of nonlinearly elastic circular rings," Meccanica, vol. 50, no. 3, pp. 689-705, 2015.

[8] C. T. Loy, K. Y. Lam, and J. N. Reddy, "Vibration of functionally graded cylindrical shells," International Journal of Mechanical Sciences, vol. 41, no. 3, pp. 309-324, 1999.

[9] L. Li and D. G. Zhang, "Free vibration analysis of rotating functionally graded rectangular plates," Composite Structures, vol. 136, no. 2, pp. 493-504, 2016.

[10] A. Gupta, M. Talha, and B. N. Singh, "Vibration characteristics of functionally graded material plate with various boundary constraints using higher order shear deformation theory," Composites Part B: Engineering, vol. 94, no. 7, pp. 64-74, 2016.

[11] C. P. Filipich, M. T. Piovan, J. M. Ramirez, and S. Domini, "A model for forced vibrations of ceramic/metallic thick rings," International Journal of Engineering Science, vol. 49, no. 10, pp. 1095-1111, 2011.

[12] H. M. Wang and D. S. Luo, "Exact analysis of radial vibration of functionally graded piezoelectric ring transducers resting on elastic foundation," Applied Mathematical Modelling, vol. 40, no. 4, pp. 2549-2559, 2016.

[13] J. Kirkhope, "Simple frequency expression for the in-plane vibration of thick circular rings," Journal of the Acoustical Society of America, vol. 59, no. 1, pp. 86-89, 1976. 
[14] T. Y. Chen and H. S. Shen, Buckling of Structures, ShangHai Scientific and Technological Literature Publishing House Co. Ltd., Shanghai, China, 1993.

[15] M. Kerdegarbakhsh, Y. Kiani, S. E. Esfahani, and M. R. Eslami, "Postbuckling of FGM rings," International Journal of Mechanical Sciences, vol. 85, pp. 187-195, 2014. 


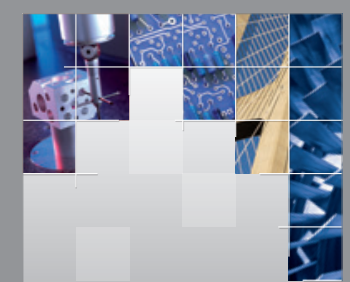

\section{Enfincering}
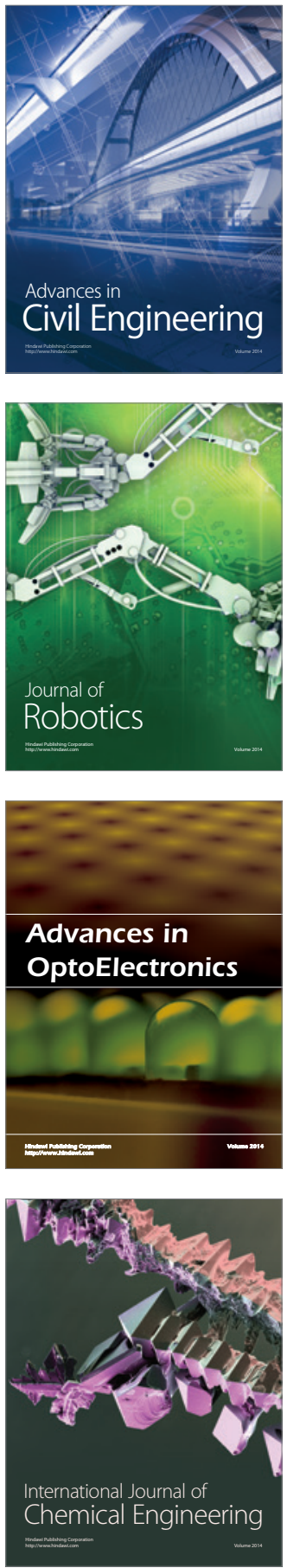

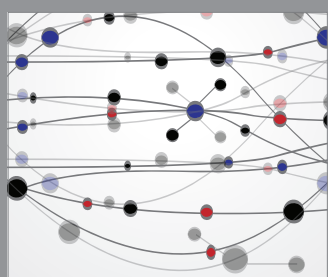

The Scientific World Journal

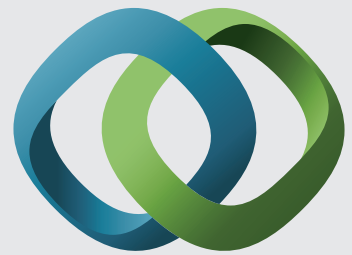

\section{Hindawi}

Submit your manuscripts at

https://www.hindawi.com
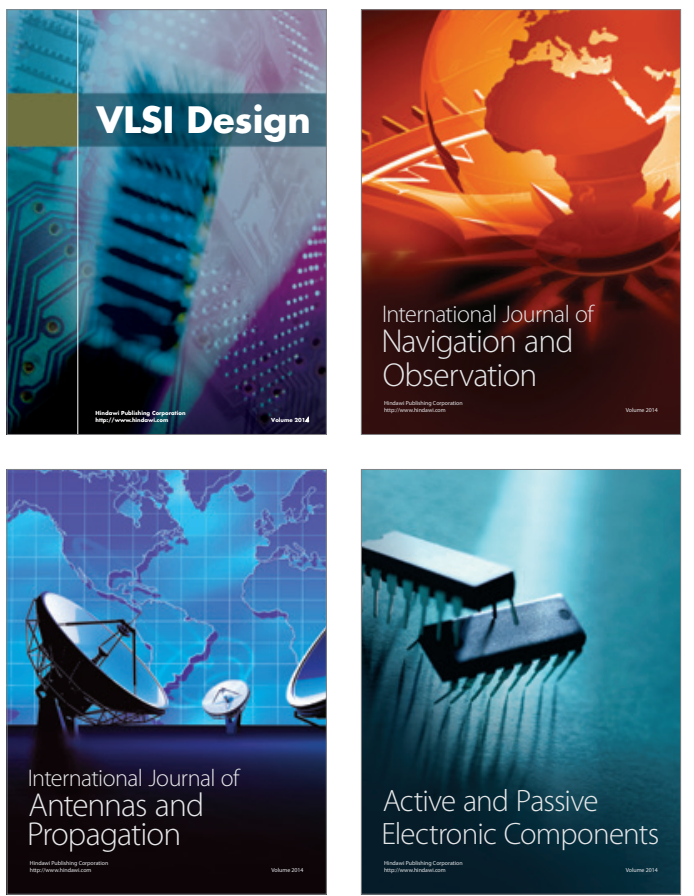
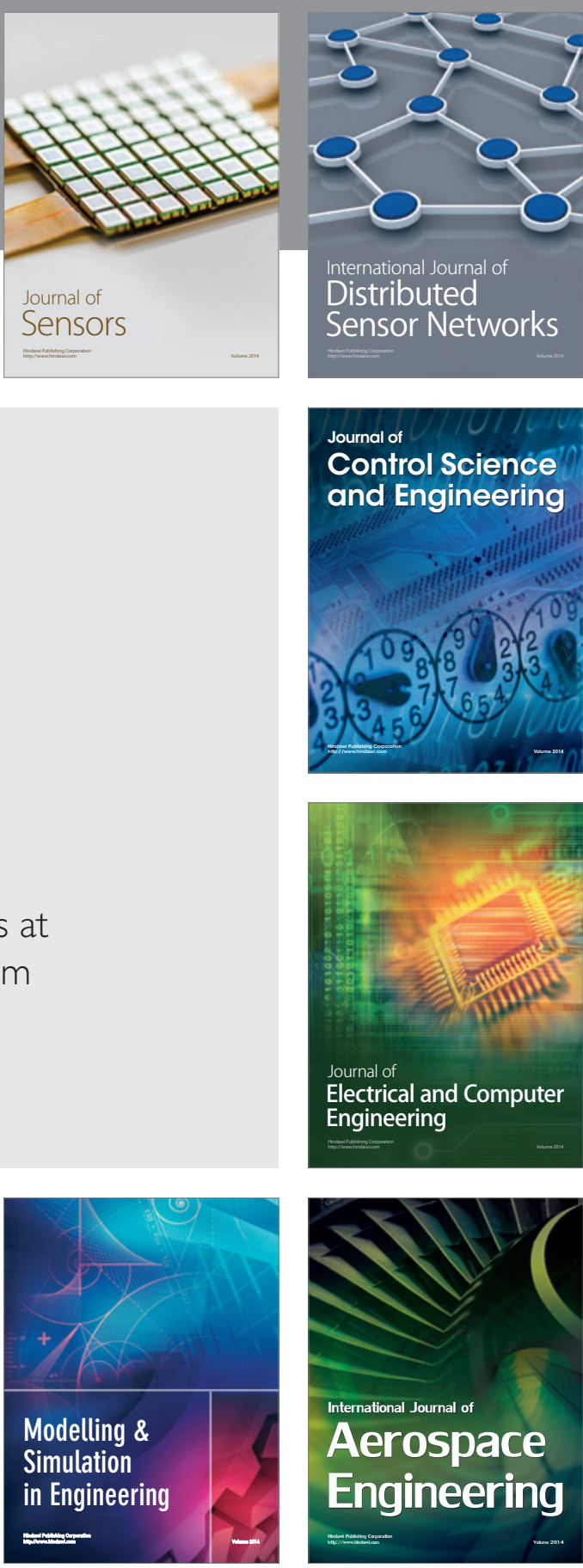

International Journal of

Distributed

Sensor Networks

$-$

Joumal of

Control Science

and Engineering
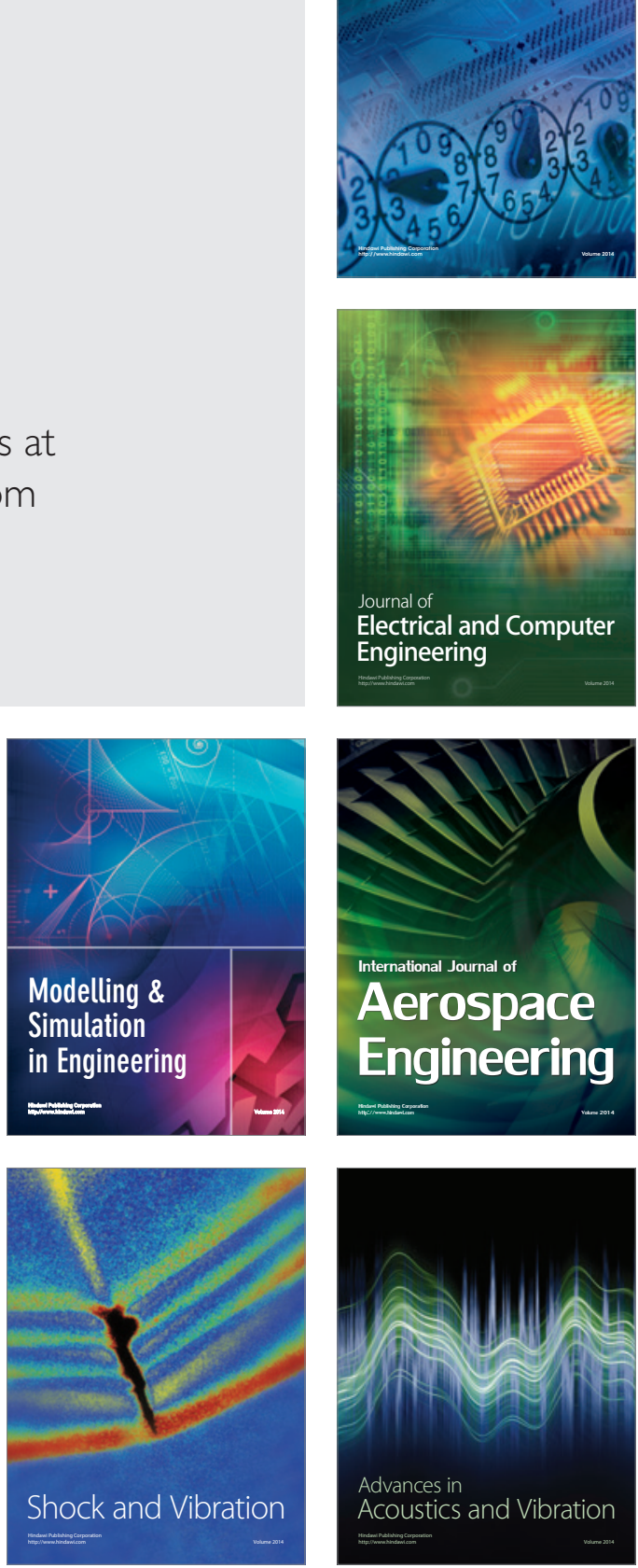